\title{
Differential down-modulation of HLA class I and II molecule expression on human tumor cell lines upon in vivo transfer
}

\author{
Riccardo Turrini $\cdot$ Anna Merlo $\cdot$ Riccardo Dolcetti $\cdot$ \\ Paola Zanovello $\cdot$ Antonio Rosato
}

Received: 7 October 2010 / Accepted: 20 July 2011 / Published online: 11 August 2011

(C) The Author(s) 2011. This article is published with open access at Springerlink.com

\begin{abstract}
Previous evidence from our laboratory showed that Epstein-Barr virus-immortalized lymphoblastoid B cells undergo a prominent down-modulation of HLA-II molecule expression when injected intraperitoneally in SCID mice, while HLA-I remains almost unaffected. Since this phenomenon can alter the experimental outcome of therapeutic protocols of adoptive cell therapy, we decided to evaluate the behavior of MHC antigens in a panel of cell lines belonging to the $\mathrm{B}$ - and T-cell lineages, as well as in epithelial tumor cell lines. Cells were administered in mice either intraperitoneally or subcutaneously and recovered 4 days later for HLA molecule expression analysis. Collected data showed a highly heterogeneous in vivo behavior of the various cell lines, which could alternatively downmodulate, completely abrogate or maintain unchanged the expression of either MHC-I or MHC-II molecules. Moreover, the site of injection impacted differentially on these
\end{abstract}

Riccardo Turrini and Anna Merlo contributed equally to the work.

Electronic supplementary material The online version of this article (doi:10.1007/s00262-011-1086-3) contains supplementary material, which is available to authorized users.

R. Turrini $\cdot$ A. Merlo $\cdot$ P. Zanovello $\cdot$ A. Rosato $(\varangle)$ Department of Oncology and Surgical Sciences,

University of Padova, Via Gattamelata, 64, 35128 Padova, Italy

e-mail: antonio.rosato@unipd.it

R. Dolcetti

Cancer Bio-Immunotherapy Unit,

Department of Medical Oncology, CRO-IRCCS,

National Cancer Institute, Aviano, PN, Italy

P. Zanovello $\cdot$ A. Rosato

Istituto Oncologico Veneto IRCCS, Padova, Italy aspects. Although such phenomena still lack a comprehensive clarification, epigenetic mechanisms are likely to be involved as epigenetic drugs could partially counteract MHC down-modulation in vivo. Nonetheless, it has to be pointed out that careful attention must be paid to the assessment of therapeutic efficacy of translational protocols of adoptive immunotherapy, as modulation of MHC molecules on human target cells when transferred in a mouse environment could readily interfere with the desired and expected therapeutic effects.

Keywords HLA-I · HLA-II $\cdot$ Mouse model ·

Down-modulation

\section{Introduction}

The use of pre-clinical mouse models represents a common approach exploited to validate laboratory observations in vivo. These models allow to study disease states in ways that would be unfeasible in human patients or that would imply harm to be inflicted on humans.

In particular, the use of immunosuppressed mice inoculated with human tumor cell lines is widely exploited in tumor immunology, where they are used to test the effects of different therapeutic approaches, including protocols of adoptive cell therapy (ACT). In this regard, we previously reported results of pre-clinical ACT immunotherapy employing cytolytic $\mathrm{CD} 4^{+} \mathrm{T}$ cells directed to Epstein-Barr virus (EBV) antigens [1]. While these populations exerted a very relevant in vitro MHC-II-restricted cytotoxic activity against autologous EBV-transformed lymphoblastoid cell lines (LCL), nonetheless they disclosed a reduced in vivo efficacy in comparison with their $\mathrm{CD} 8^{+} \mathrm{EBV}$-specific counterpart. We found that expression of HLA-II molecules on 
LCL underwent a rapid down-modulation upon in vivo transfer. This phenomenon could be reverted by the in vivo use of a demethylating agent (decitabine) that significantly restored the HLA-II expression on LCL [1].

Here, we extended the analysis of the expression of MHC antigens on a panel of different cell lines after ex vivo recovery following either subcutaneous (s.c.) or intraperitoneal (i.p.) administration, as such markers are critically involved in the interaction between effector and target cells, and therefore, their modulation could strongly impact on the results of the experimental approaches of ACT.

Our results indicate that different cell lines behave differently and that even the site of injection can influence the expression of HLA molecules in vivo. Since the expression of MHC-I and MHC-II can be under epigenetic control [2], we assessed these aspects in our model using epigenetic drugs in vivo. Here, we provide evidence that one of the potential reasons for the observed down-modulation relies on epigenetic mechanisms, likely activated by the mouse microenvironment.

Based on these observations, we suggest that any ACT approach involving human tumor cell lines transferred in a mouse environment should be preceded by a careful analysis of MHC expression along tumor growth in vivo, since HLA antigen down-modulation could introduce a strong bias in the assessment of therapeutic efficacy of adoptively transferred effector $\mathrm{T}$ cells.

\section{Materials and methods}

\section{Cell lines}

The following human cell lines were used: DG-75, Daudi, Namalwa, and BL-41 are Burkitt lymphoma B cell lines; BL-41 B95.8 is the EBV-infected counterpart cell line; Raji is a lymphoblast-like cell line derived from a Burkitt's lymphoma; LCL is a lymphoblastoid cell line generated by EBV infection of PBMC; SH-9 is a lymphoblastoid cell line that spontaneously immortalized and derived from a Hodgkin's lymphoma patient; HuT-78 and Jurkat are T cell lymphoma cell lines; MOLT-3 and CEM are acute lymphoblastic leukemia T cell lines; IGROV-1 and OVCAR-3 are ovary adenocarcinoma cell lines; SK23-Mel, UACC-257, and A375 are malignant melanoma cell lines.

\section{In vivo experiments}

Six- to 8-week-old female SCID mice were purchased from Charles River Laboratories (Calco, Italy) and housed in our specific pathogen-free animal facility. Procedures involving animals and their care were in conformity with institutional guidelines (D.L. 116/92 and subsequent complementing circulars), and all experimental protocols were approved by the local ethical committee of Padova University (CEASA).

A total of $80-100 \times 10^{6}$ cells from every cell line were injected i.p., while $20-25 \times 10^{6}$ cells were used for s.c. injection. Four days later, the subcutaneous masses were recovered, mechanically disaggregated, and used for immunophenotyping. Similarly, i.p. injected cells were recovered by peritoneal washing and used for the flow cytometry analyses. A high number of cells were required for the initial injection to provide the recovery of sufficient cells for subsequent staining and in vitro culture.

In vitro and in vivo treatment with epigenetic drugs

Tumor cells explanted ex vivo were cultured in vitro with different epigenetic drugs (trichostatin A and decitabine, also known as $2^{\prime}$-deoxy-5-azacytidine) that had already proven to be effective in vitro [3,4] or in vivo [1]. Cells were plated at $5 \times 10^{5}$ cells/well in 24-well plate in complete medium (control) or medium added with trichostatin A $(50,250$, and $500 \mathrm{nM}$, Sigma $)$ or decitabine $(1 \mu \mathrm{M}$, Pharmachemie B.V., Haarlem, Holland), for up to 72 h. For in vivo experiments, mice were treated with trichostatin $\mathrm{A}$ ( $1 \mathrm{mg} / \mathrm{kg}$ once a day, days 0-3, Sigma [5]), decitabine $(0.25 \mathrm{mg} / \mathrm{kg}$ once at day 0 , then twice a day, days $1-3$ [1]), suberoylanilide hydroxamic acid (SAHA, $1.25 \mathrm{mg} / \mathrm{mouse}$, once a day, days 1-3, Alexis Biochemicals, Lausen, Switzerland [6]), valproic acid (5 $\mathrm{mg} /$ mouse, once a day, days $1-3$, modified from [7], Sigma), and Interferon- $\gamma$ (50,000 IU/mouse, once, $6 \mathrm{~h}$ before cells recovery, modified from [1], PeproTech, Rocky Hill, NJ). On day 4, cells were recovered by peritoneal washing and used for the flow cytometry analyses.

Flow cytometry analysis

Human cancer cells recovered ex vivo were initially gated according to their forward- and side-scatter profiles and then specifically identified for their human origin with antibodies to CD5 (for the MOLT-3 cell line; clone MEM-32, ImmunoTools, Friesoythe, Germany), CD19 (for all the B cell lines; clone 89B, Coulter, Milano, Italy), CD45 (for all the $\mathrm{T}$ cell lines, but MOLT-3; clone MEM-28, ImmunoTools), and CD44 antibody (for all the epithelial cancer cell lines; clone J-173, Immunotech Coulter, Milano, Italy). The gated populations always confirmed to be $100 \%$ positive for the respective markers.

Further stainings were performed with antibodies to B2-microglobulin (clone 2M2, BioLegend, Cambridge, UK), HLA-I (HLA-A, HLA-B, and HLA-C loci, clone W6/32, BioLegend), HLA-A2 (clone BB7.2, BioLegend), HLA-II (clone Tü39, BD, Erembodegem, Belgium), HLA-DR (clone L243, BD; clone LN3, eBiosciences, 
San Diego, California, USA), HLA-DQ (clone HLADQ1, BioLegend; clone Tu169, BD), and PAN-Ig (Goat polyclonal anti-human IgG, IgA, and IgM $(\mathrm{H}+\mathrm{L})$, AbD Serotec, Oxford, UK). Control stainings were performed using the appropriate isotypes (mouse IgG1 for $\beta 2$-microglobulin and HLA-DQ, BD; goat IgG for PAN-Ig, AbD Serotec; mouse $\operatorname{IgG} 2 \mathrm{a}$ for all the other antibodies, BD).

To avoid potential bias in recognizing the implanted human cell lines, all antibodies were tested for cross-reactivity with mouse splenocytes and found negative.

After staining, red blood cells were lysed and cells were analyzed using FACSCalibur (BD) instrument and FlowJo software (TreeStar Inc., Olten, Switzerland).

Percentage of positivity was calculated as the difference between the positivity percentage of each marker and that of the corresponding isotype control.

\section{Results}

Based on our previous results [1], first we decided to extend the assessment of MHC-I and MHC-II expression, before in vivo administration and after ex vivo recovery, to a panel of B cell lines. Such analysis revealed that in all cases the percentage of positivity and the mean fluorescence intensity (MFI) of samples were lower after ex vivo evaluation, but no general rule could be inferred. DG-75, SH-9, Raji (this latter being reported in Fig. 1a, b as an example of this group of cells), and Daudi (which does not harbor MHC class I) cell lines completely or partially down-modulated HLA-I and HLA-II molecule expression when injected i.p. On the other hand, most of the cell lines maintained a high expression of the analyzed markers when injected s.c., with the exception of LCL and, partially, SH9, Namalwa, and Daudi cell lines (LCL and Namalwa illustrate this behavior in Fig. 1b). The only B cell lines that preserved the expression of MHC-I and MHC-II markers, after both i.p. and s.c. injection, were BL-41 and their EBV-infected counterpart (Fig. 1a, b). Expression data obtained with pan anti-HLA-I were fully confirmed by staining recovered cells with anti$\beta 2$-microglobulin antibody (Fig. 1b) and anti-HLA-A2 allele-specific antibody (where present, data not shown). Similarly, results of cytometry analyses with the use of anti-HLA-DR and anti-HLA-DQ antibodies completely overlapped those obtained with pan anti-HLA-II (data not shown).

Interestingly, even the expression of surface immunoglobulins underwent down-modulation when injected i.p. (especially in Raji cell line), while it was generally maintained after s.c. injection (Fig. 1b).

When $\mathrm{T}$ cells were considered (Fig. 2a), it turned out that the s.c. injection induced the partial down-modulation of MHC-I on all the lines we studied, except MOLT-3. The i.p. inoculation route affected marker expression on MOLT3 and HuT-78 cells but did not influence Jurkat and CEM cell lines. Finally, two ovarian carcinoma and three malignant melanoma cell lines were tested: IGROV-1 and OVCAR-3 tumors strongly down-modulated HLA-I expression after either i.p. or s.c. injection, while all melanomas retained MHC-I expression when injected i.p. but lost it after s.c. administration. (Fig. 2b shows only SK23$\mathrm{Mel}$, as an example of the behavior of the three melanoma cell lines.) Again, also in the case of both T cell and epithelial tumor cell lines, data of pan MHC-I and MHC-II expression were confirmed by staining with anti- $\beta 2$-microglobulin and allele-specific antibodies (Fig. 2a, b and data not shown).

As some HLA-I and HLA-II promoters (and their transactivators) can be under epigenetic control [2], host and tumor microenvironments might induce methylation and histone deacetylation in tumor cells, thus affecting the expression of the MHC-I and MHC-II molecules [8, 9]. To evaluate this possibility, we cultured tumor cells explanted ex vivo with different epigenetic drugs (namely, trichostatin A and decitabine) that had already proven to be effective in vitro [3, 4] or in vivo [1], respectively. Surprisingly, we observed that the simple placement of tumor cells in complete medium, without the addition of any drug, led to reversion of the down-modulated phenotype (when present) within a few hours (data not shown). To better clarify this issue, we decided to test the different epigenetic drugs in vivo, in parallel with interferon- $\gamma$, a well-known inducer of MHC molecule expression. Mice were injected i.p. with tumor cell lines and treated with trichostatin A, decitabine, suberoylanilide hydroxamic acid, valproic acid, or Interferon- $\gamma$. As it can be appreciated in online resource, Supplemental Figure, decitabine positively modulated HLA-II and HLA-I expression on LCL cells, while the treatment with trichostatin A partially restored the MHC-I expression on the IGROV-1 cell line.

\section{Discussion}

Adoptive cell therapy is regarded as an efficient and promising approach for the treatment of different tumors, ranging from several EBV-related malignancies $[1,10]$ to melanoma [11]. Before a new ACT protocol is accepted for clinical use, its efficacy has to be demonstrated in animal models, mainly in mice injected with tumor cell lines to simulate the disease. While these models are generally accepted, notwithstanding they raise concerns regarding the cytokine milieu, the tumor microenvironment, the inoculation setting, and the life span of human-origin components that are transferred into the mouse, which can give rise to several biases ultimately perturbing the outcome $[12,13]$. 
Fig. 1 a Representative cytofluorimetric analysis of DG-75, BL-41 B95.8, and Raji cell lines: expression of different markers (HLA-I for DG-75 and BL-41 B95.8 cells and HLA-II for Raji cell line) after i.p. (dotted line) or s.c. (dashed line) recovery in comparison with in vitro expression (black line). Gray areas represent the isotype controls. b HLA-I, $\beta 2$-microglobulin (b2m), HLA-II, and immunoglobulins (Ig) percentage of positivity (left panels) and MFI of expression (right panels) from selected B cell lines after i.p. and s.c. injection. White and black bars refer to values of cells recovered from i.p. and s.c. sites, respectively, while gray bars show data of cells maintained in in vitro culture. The percentage of positivity for in vitro markers expression is not shown and is always $100 \%$. Figure shows mean \pm SD of at least 3 independent experiments for each cell line
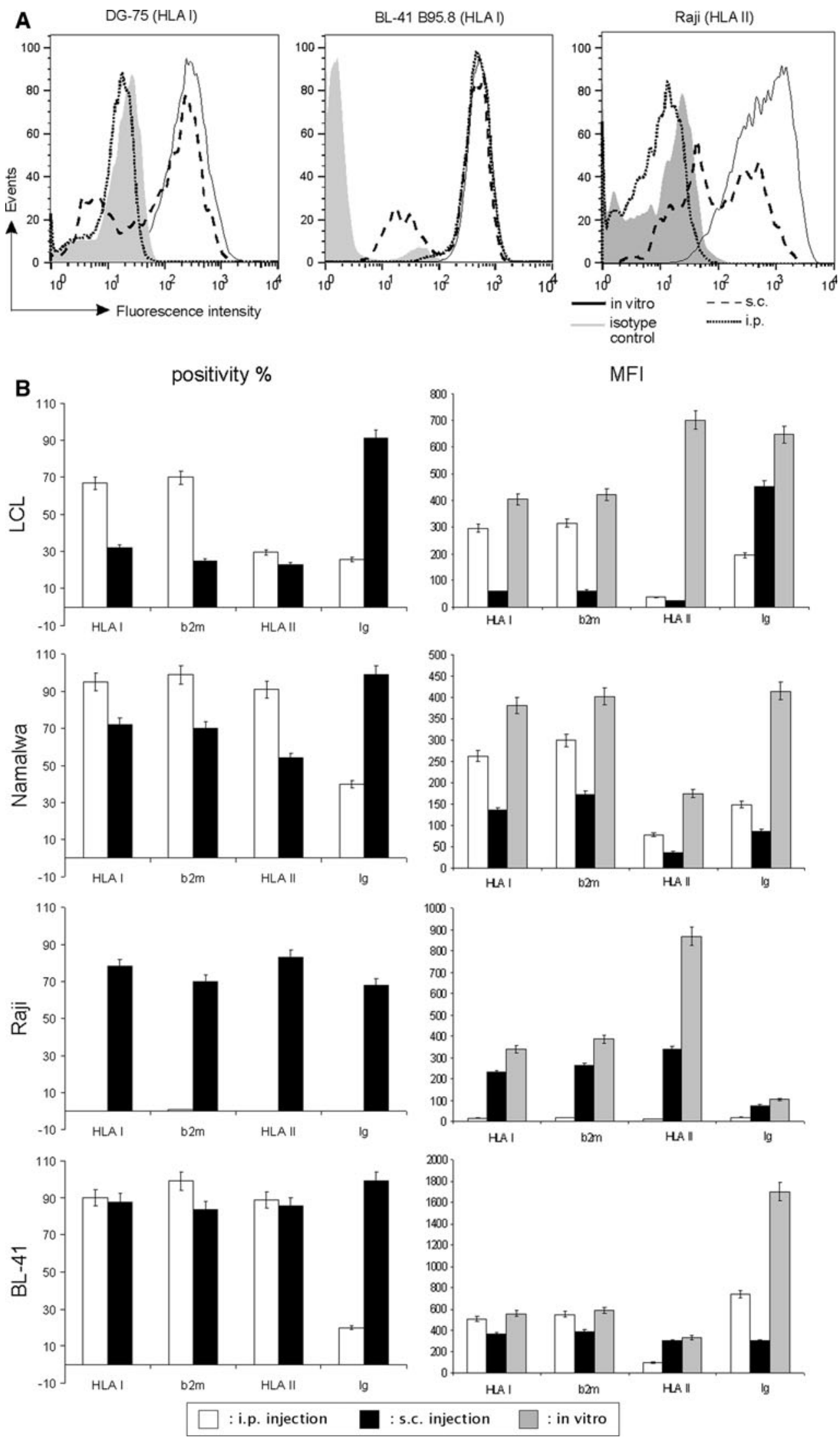
Fig. 2 a HLA-I, $\beta 2$-microglobulin (b2m), and HLA-II percentage of positivity and MFI of expression on different $\mathrm{T}$ cell lines after i.p. and s.c. recovery. White and black bars refer to i.p. and s.c. injection, respectively, while gray bars refer to the in vitro condition. The percentage of positivity for in vitro markers expression is not shown and is always $100 \%$. Figure shows mean \pm SD of at least 3 independent experiments for each cell line. b Cytofluorimetric analysis of different epithelial tumor cell lines. Bars as in

a. Figure shows mean $\pm \mathrm{SD}$ of at least 3 independent experiments for each cell line
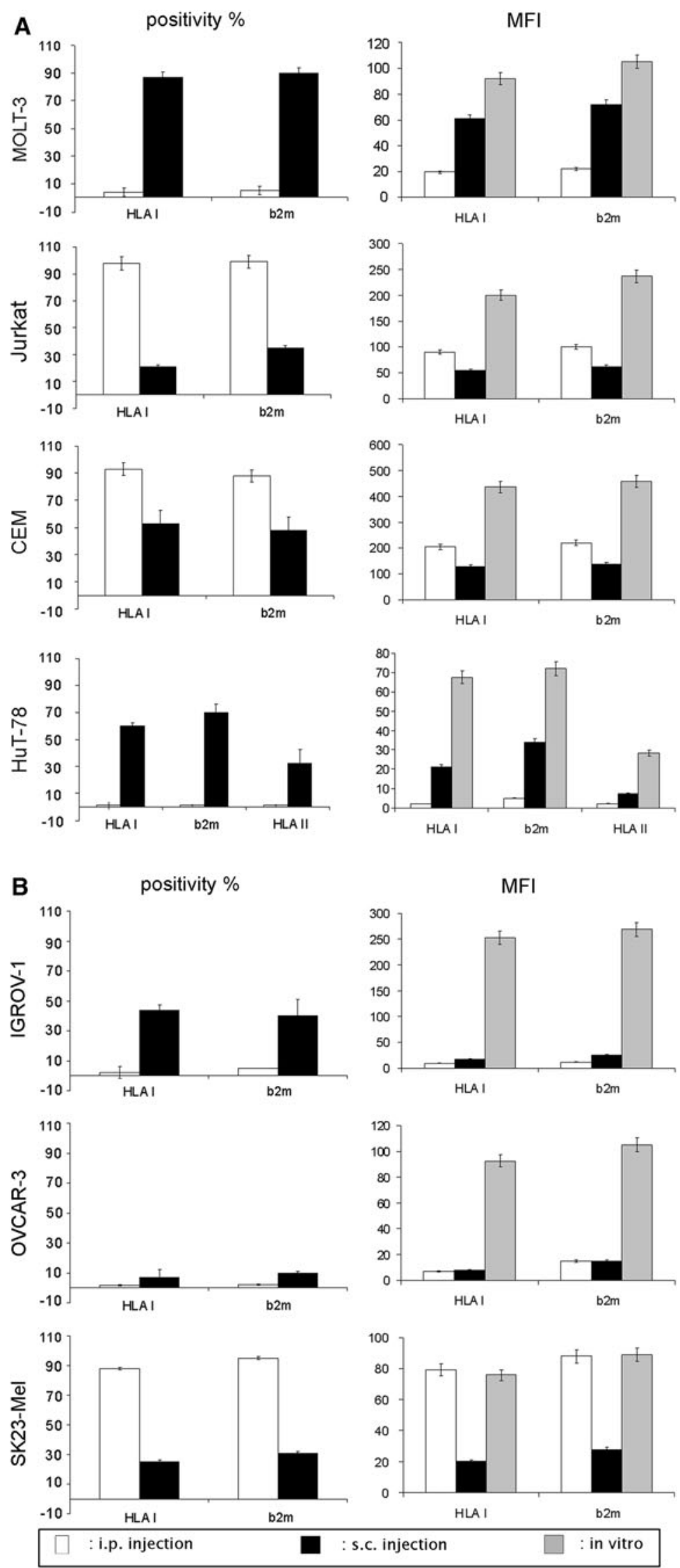
An example of such aspects can be found in a previous work from our group [1] where we assessed the therapeutic potentiality of EBV-specific $\mathrm{CD} 4^{+}$cytotoxic $\mathrm{T}$ cells, which were effective in vitro, but not in vivo. We observed that LCL target cells, when injected in SCID mice, underwent a rapid HLA-II down-modulation, thus reducing the natural recognition of MHC-II peptide complexes by $\mathrm{CD} 4^{+}$CTL. Since this behavior was not observed in humans [1], we concluded it was induced in the mouse model by some unidentified stimuli that could be partially overcome by using demethylating agents [1]. Based on such observation, we wondered whether this phenomenon was limited to LCL or could represent a more frequent occurrence. Therefore, we first extended the panel of B cell lines tested that were analyzed 4 days after injection, because previous results showed that the maximal down-modulation was already reached at this time point [1] and maintained afterward (data not shown): results indicated that a recurrent behavior could not be established. Moreover, the site of injection had a profound influence on the observed down-modulation. In particular, with the exception of Namalwa, BL-41, and BL41 B95.8 cell lines, all the other B cell lines down-modulated the expression of HLA-I and/or HLA-II when injected i.p., while the s.c. inoculation negatively impacted only on LCL, SH9, Daudi, and partially on Namalwa cell lines. Notably, surface immunoglobulin expression was also reduced in some cell lines and in a differential manner according to the injection site, when recovered ex vivo in comparison with in vitro culture.

Since no general rule could be extrapolated, we expanded our analysis to some $\mathrm{T}$ cell lines: again, no coherent behavior could be identified as HLA-I expression was totally abrogated in MOLT-3 and HuT-78, but not in Jurkat and CEM cell lines when injected intraperitoneally; the s.c. injection induced a partial down-modulation on all $\mathrm{T}$ cell lines, but the MOLT-3 cells. Finally, MHC class I expression on some epithelial tumor cell lines appeared undergoing a constant down-modulation, but even in this case with an exception represented by melanomas after i.p. injection.

Whereas the causes of such phenomena are not fully clarified yet, nonetheless epigenetic mechanisms are likely to be involved, as supported by results we reported with epigenetic drugs; indeed, decitabine partially restored MHC-II expression in LCL cells, thus indicating that the methylation of promoters of genetic elements involved in the expression of HLA alleles may play an important role in MHC down-modulation [1]. On the other hand, trichostatin A counteracted HLA down-modulation in IGROV-1 cells, a finding suggesting that histone acetylation may represent an additional relevant mechanism, as previously reported in a different setting [3]. These data are also in line with a previous study reporting an epigenetic effect of the microenvironment, where bystander stromal cells may have a major influence on the growth and progression of tumors [14]. Moreover, in both the peritoneal cavity and, more pronouncedly, the inner mass of subcutaneous inoculi, tumor cells might undergo an hypoxic state, which has been already demonstrated to down-modulate HLA-G and MHC-I-related chain A (MICA) [15, 16]; further, hypoxia has been also directly involved in MHC-I and MHC-II down-modulation in rat cells [17].

In conclusion, no general rule regarding the maintenance or down-modulation of MHC-I or MHC-II molecules on human tumor cell lines upon in vivo transfer in immunodeficient mice can be inferred by our data; indeed, every cell line appears to possess a particular behavior. These observations raise some important concerns about the interpretation of data resulting from the administration of human $\mathrm{T}$ cells into human-tumor-bearing mice, since negative results can be due to the absence of target molecules on the surface of cancer cells. Overall, our suggestion is therefore to check carefully the expression of MHC antigens on tumor cell lines upon their in vivo transfer before testing an ACT therapeutic protocol.

Acknowledgments This study was partly supported by grants from the Italian Ministry of Health (Progetto oncologico di medicina molecolare: i tumori femminili; Progetto strategico: Farmaci cellulari, vaccini e bioterapie innovative dei tumori; Alleanza Contro il Cancro, ACC-4) and the Italian Association for Cancer Research (AIRC).

Open Access This article is distributed under the terms of the Creative Commons Attribution Noncommercial License which permits any noncommercial use, distribution, and reproduction in any medium, provided the original author(s) and source are credited.

\section{References}

1. Merlo A, Turrini R, Bobisse S et al (2010) Virus-specific cytotoxic $\mathrm{CD} 4+\mathrm{T}$ cells for the treatment of EBV-related tumors. J Immunol. doi:10.4049/jimmunol.0902850

2. Wright KL, Ting JP (2006) Epigenetic regulation of MHC-II and CIITA genes. Trends Immunol 27:405-412. doi:10.1016/j.it. 2006.07.007

3. Paco L, Garcia-Lora AM, Casares C et al (2007) Total loss of HLA class I expression on a melanoma cell line after growth in nude mice in absence of autologous antitumor immune response. Int $\mathrm{J}$ Cancer 121:2023-2030. doi:10.1002/ijc.22925

4. Garrido C, Algarra I, Maleno I et al (2010) Alterations of HLA class I expression in human melanoma xenografts in immunodeficient mice occur frequently and are associated with higher tumorigenicity. Cancer Immunol Immunother 59:13-26. doi:10.1007/ s00262-009-0716-5

5. Tao R, de Zoeten EF, Özkaynak E et al (2007) Deacetylase inhibition promotes the generation and function of regulatory $\mathrm{T}$ cells. Nat Med 13:1299-1307. doi:10.1038/nm1652

6. Neureiter D, Zopf S, Leu T et al (2007) Apoptosis, proliferation and differentiation patterns are influenced by Zebularine and SAHA in pancreatic cancer models. Scand J Gastroenterol 42:103-116. doi:10.1080/00365520600874198

7. Li XN, Shu Q, Su JMF, Perlaky L, Blaney SM, Lau CC (2005) Valproic acid induces growth arrest, apoptosis, and senescence in 
medulloblastomas by increasing histone hyperacetylation and regulating expression of p21Cip1, CDK4, and CMYC. Mol Cancer Ther 4:1912. doi:10.1158/1535-7163.MCT-05-0184

8. Seliger B, Maeurer MJ, Ferrone S (2000) Antigen-processing machinery breakdown and tumor growth. Immunol Today 21:455-464

9. Seliger B, Wollscheid U, Momburg F, Blankenstein T, Huber C (2001) Characterization of the major histocompatibility complex class I deficiencies in B16 melanoma cells. Cancer Res 61:10951099. doi:10.1016/S0167-5699(00)01692-3

10. Merlo A, Turrini R, Dolcetti R et al (2010) The interplay between Epstein-Barr virus and the immune system: a rationale for adoptive cell therapy of EBV-related disorders. Haematologica 95:1769-1777. doi:10.3324/haematol.2010.023689

11. Bobisse S, Rondina M, Merlo A et al (2009) Reprogramming T lymphocytes for melanoma adoptive immunotherapy by $\mathrm{T}$-cell receptor gene transfer with lentiviral vectors. Cancer Res 69: 9385-9394. doi:10.1158/0008-5472.CAN-09-0494
12. de Jong M, Maina T (2010) Of mice and humans: are they the same? - Implications in cancer translational research. J Nucl Med 51:501-504. doi:10.2967/jnumed.109.065706

13. Wall RJ, Shani M (2008) Are animal models as good as we think? Theriogenology 69:2-9. doi:10.1016/j.theriogenology.2007.09.030

14. Tlsty TD, Coussens LM (2006) Tumor stroma and regulation of cancer development. Annu Rev Pathol 1:119-150. doi:10.1146/ annurev.pathol.1.110304.100224

15. Mouillot G, Marcou C, Zidi I et al (2007) Hypoxia modulates HLA-G gene expression in tumor cells. Hum Immunol 68:277285. doi:10.1016/j.humimm.2006.10.016

16. Luo L, Lu J, Wei L et al (2010) The role of HIF-1 in up-regulating MICA expression on human renal proximal tubular epithelial cells during hypoxia/reoxygenation. BMC Cell Biol 11:91. doi:10.1186/ 1471-2121-11-91

17. Eisenberger CF, Viebahn R, Lauchart W, de Groot H, Becker HD (1994) MHC antigen presentation on the surface of hepatocytes: modulation during and after hypoxic stress. Transpl Int 7(1):S163-S166 